\title{
Expanding the pathologic spectrum of light chain deposition disease: a rare variant with clinical follow-up of 7 years
}

\author{
Anthony Chang ${ }^{1}$, Carine J Peutz-Kootstra ${ }^{2}$, Catherine A Richardson ${ }^{3}$ and Charles E Alpers ${ }^{1}$ \\ ${ }^{1}$ Department of Pathology, University of Washington Medical Center, Seattle, WA, USA; ${ }^{2}$ Department of \\ Pathology, Academic Hospital Maastricht, Maastricht, The Netherlands and ${ }^{3}$ Pacific Nephrology Associates, \\ Tacoma, WA, USA
}

\begin{abstract}
We report an unusual histologic manifestation of light chain deposition disease in a 69-year-old female patient, who presented with nephrotic syndrome and an increased serum creatinine. The renal biopsy findings by light and electron microscopy suggested a glomerulonephritis with massive immune-complex deposition, such as lupus nephritis. However, the overall clinical scenario was inconsistent with lupus. Subsequent tests revealed multiple myeloma confirmed by bone marrow biopsy and identification of a monoclonal kappa light chain immunoglobulin by serum and urine immunoelectrophoresis and immunofixation. Additional immunohistochemistry of the first biopsy also demonstrated strong kappa light chain staining of the glomerular capillary walls and mesangium but not lambda light chain or IgG staining. The patient responded well to therapy and was asymptomatic until nearly 7 years later. A repeat biopsy revealed similar findings to the first biopsy with the addition of immunofluorescence microscopy, which confirmed the prominent kappa light chain staining of the glomeruli, tubular basement membranes, and interstitium with corresponding electron-dense deposits visualized by electron microscopy. This case represents an unusual histologic variant of light chain deposition disease, which to our knowledge has not been previously described and further expands the wide clinicopathologic spectrum within the diagnostic entity of light chain deposition disease.
\end{abstract}

Modern Pathology (2005) 18, 998-1004. doi:10.1038/modpathol.3800368; Published online 14 January 2005

Keywords: light chain deposition disease; monoclonal immunoglobulin deposition disease; multiple myeloma

Light chain deposition disease has been found in $10 \%$ of renal biopsies of patients with an underlying monoclonal gammopathy. ${ }^{1}$ Patients with this disease may have clinical presentations of proteinuria, renal insufficiency, or both. The histologic features are frequently characterized by nodular glomerulosclerosis, but a wide spectrum of glomerular changes has been described which ranges from an absence of histologic changes by light microscopy, indistinguishable from minimal change disease, to variable degrees of mesangial alterations, to membranoproliferative glomerulonephritis. ${ }^{2-9}$ Immunofluorescence microscopy or immunohistochemical localization is essential for establishing the diagnosis of light chain deposition disease, which is characterized by monoclonal deposition of either

Correspondence: Dr A Chang, MD, University of Chicago Hospitals, 5841 S Maryland Ave., Chicago, IL 60637, USA

E-mail: anthony.chang@uchospitals.edu

Received 2 August 2004; revised and accepted 29 November 2004; published online 14 January 2005 kappa or lambda light chains, but not both, in glomerular and tubular basement membranes, mesangium, interstitium, and vessel walls. Electron microscopy typically demonstrates granular electron-dense deposits, often with a confluent appearance, involving the glomerular and tubular basement membranes.

In this report, we present a rare variant of light chain deposition disease with histologic features that mimic a glomerulonephritis with massive immune-complex deposition. Cases with similar ultrastructural features but with deposits of monoclonal immunoglobulins have been recently described and have been proposed to comprise a unique clinicopathologic entity. ${ }^{10}$ An alternate perspective suggested by our case is that cases of monoclonal immunoglobulin deposition and predominantly light chain deposition disease with ultrastructural features of immune-complex glomerulonephritis represent an overlapping clinical and pathologic spectrum, similar to what has previously been encountered in other glomerulopathies 
associated with lymphoplasmacytic disorders. A particular example of such an overlapping pattern of renal injury would be nodular glomerulosclerosis, which can be encountered in heavy chain deposition disease (no or minimal light chain deposits present), monoclonal immunoglobulin deposition disease (both light and heavy immunoglobulin chain deposits present), and light chain deposition disease (no immunoglobulin heavy chain present). Despite the unique histologic and ultrastructural features of the case presented below, the corresponding clinical features fall within the reported experience of light chain deposition disease and suggest that our case expands the pathologic spectrum of this diagnostic category rather than representing a new diagnostic entity.

\section{Materials and methods}

Standard procedures were used to process formalinfixed tissue for light microscopic evaluation. The sections were approximately $2 \mu \mathrm{m}$ in thickness. Hematoxylin and eosin, periodic acid Schiff, and Jones methenamine silver stains on three level sections and a Masson trichrome stain on one level section were obtained for both biopsy specimens.

Owing to the absence of diagnostic glomeruli in the tissue submitted for immunofluorescence microscopy, immunohistochemistry was performed on the formalin-fixed and paraffin-embedded tissue sections of the first biopsy. We used the protocol published on the Renal Pathology Society website (http://www.renalpathsoc.org/000R000R.htm). The sections were counterstained with hematoxylin. A limited antibody panel consisting of IgG, kappa light chain, and lambda light chain (DAKO Corporation, Carpinteria, CA, USA) was performed.

Standard immunofluorescence microscopy was performed on $4 \mu \mathrm{m}$ frozen tissue sections of the second biopsy stained with polyclonal fluorescein isothiocyanate-conjugated antibodies to IgG, IgA, IgM, C3, C1q, kappa light chains, lambda light chains, fibrinogen, and albumin (DAKO). The immunofluorescence staining was scored on a semiquantitative scale from 0 to $4+$.

Standard procedures were applied to evaluate the renal specimens using a Philips EM410LS electron microscope. A magnification of up to $\times 38000$ was used to exclude the presence of substructural organization within the electron-dense deposits. The use of the term plasma cell dyscrasia refers to monoclonal proliferations of plasma cells or plasmacytoid lymphocytes, which are frequently characterized by the secretion of a monoclonal immunoglobulin component. The term multiple myeloma is used specifically when the clinical and/or pathologic criteria have been satisfied, as enumerated by the World Health Organization Classification of Tumours of Haematopoietic and Lymphoid Tissues. ${ }^{11}$

\section{Clinical history}

This 69-year-old female patient with a past medical history of well-controlled hypertension for 20 years presented with headaches and increasing hypertension, dyspnea, orthopnea, and edema involving the face and upper and lower extremities. A serum creatinine was $3.3 \mathrm{mg} / \mathrm{dl}(442 \mu \mathrm{mol} / \mathrm{l}), 1 \mathrm{month}$ prior to her initial presentation. Her blood pressure was $221 / 115 \mathrm{mmHg}$, which decreased to $110 / 55 \mathrm{mmHg}$ with aggressive angiotensin-converting enzyme inhibitor treatment following her presentation. Laboratory data were noteworthy for: blood urea nitrogen, $70 \mathrm{mg} / \mathrm{dl}(25 \mathrm{mmol} / \mathrm{l})$; creatinine, $5.0 \mathrm{mg} / \mathrm{dl}$ (442 $\mu \mathrm{mol} / \mathrm{l})$; calcium, $8.2 \mathrm{mg} / \mathrm{dl}(2.1 \mathrm{mmol} / \mathrm{l})$; hematocrit, $23 \%$; and albumin, $2.9 \mathrm{mg} / \mathrm{dl}(29 \mathrm{~g} / \mathrm{l})$. A $24-\mathrm{h}$ urine protein excretion totaled $5.8 \mathrm{~g}$. Creatinine clearance was $5 \mathrm{ml} / \mathrm{min}(0.08 \mathrm{ml} / \mathrm{s})$. A chest X-ray showed pulmonary edema. An echocardiogram demonstrated an ejection fraction of $45-50 \%$ with mitral and tricuspid regurgitation, and pulmonary hypertension. A renal ultrasound showed normalsized right and left kidneys. Serology for hepatitis A IgG was positive, but hepatitis A IgM was negative. Serologies for hepatitis B, hepatitis C, anti-neutrophil cytoplasmic antibodies, anti-glomerular basement membrane antibodies, rheumatoid factor, and HIV were negative. C3 complement component was $62 \mathrm{mg} / \mathrm{dl}$ (normal: 75-161 mg/dl), and C4 complement component was $21 \mathrm{mg} / \mathrm{dl}$ (normal: 16-47 mg/ dl) Antinuclear antibodies were positive at 1:40 with a speckled pattern. Serum cryoglobulins were not tested. A diagnostic renal biopsy was performed.

\section{Renal biopsy \#1 pathology}

The histologic sample contained approximately 10 glomeruli per level section. The glomeruli showed massive accumulation of eosinophilic material with a confluent appearance permeating the subendothelial aspect of the glomerular basement membranes, reminiscent of massive 'wire-loop' deposits, more typical of lupus nephritis, but also involving mesangial areas (Figure 1). The eosinophilic material focally appeared to occlude the lumina of a few capillary loops. There was rare splitting of the glomerular basement membranes. The glomeruli demonstrated accentuation of the lobular architecture, but nodular glomerulosclerosis or mesangial hypercellularity was not prominent. Crescent formation or necrosis of glomerular tufts was not identified. There was diffuse interstitial fibrosis with a mild degree of tubular atrophy and associated mild interstitial mononuclear cell inflammation. The arteries showed moderate intimal sclerosis without evidence of vasculitis. The arterioles were not prominent. A Congo red stain was negative for amyloid. The tissue submitted for immunofluorescence microscopy contained renal medulla only without diagnostic glomeruli, and immunofluorescence studies were not performed. A limited panel 


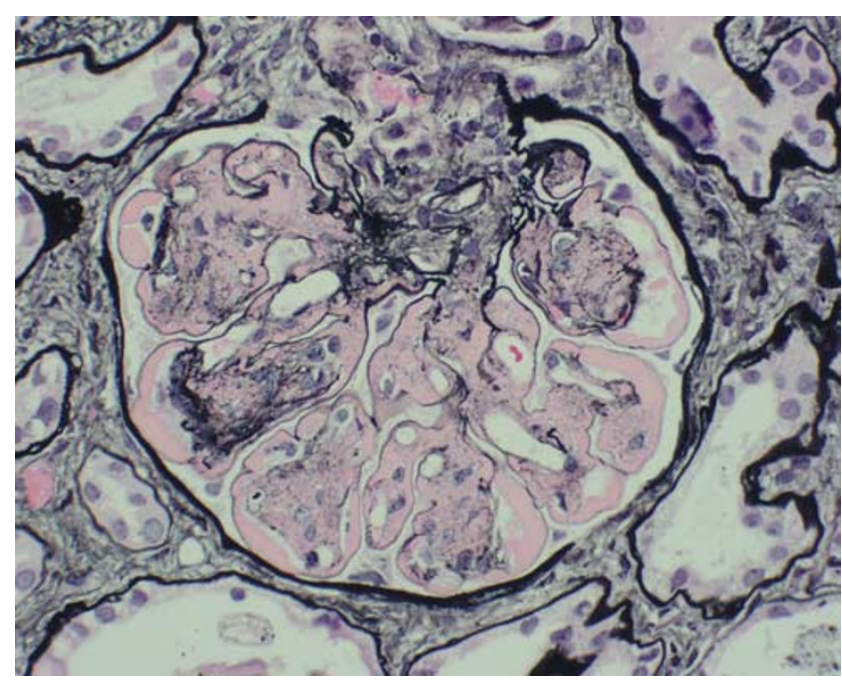

Figure 1 First biopsy. This high-power view of a representative glomerulus exhibits massive accumulation of amorphous eosinophilic material both in mesangial areas and along the subendothelial aspect of glomerular basement membranes, indistinguishable from massive 'wire-loop' type deposits that can be encountered in lupus nephritis (Jones silver methenamine).

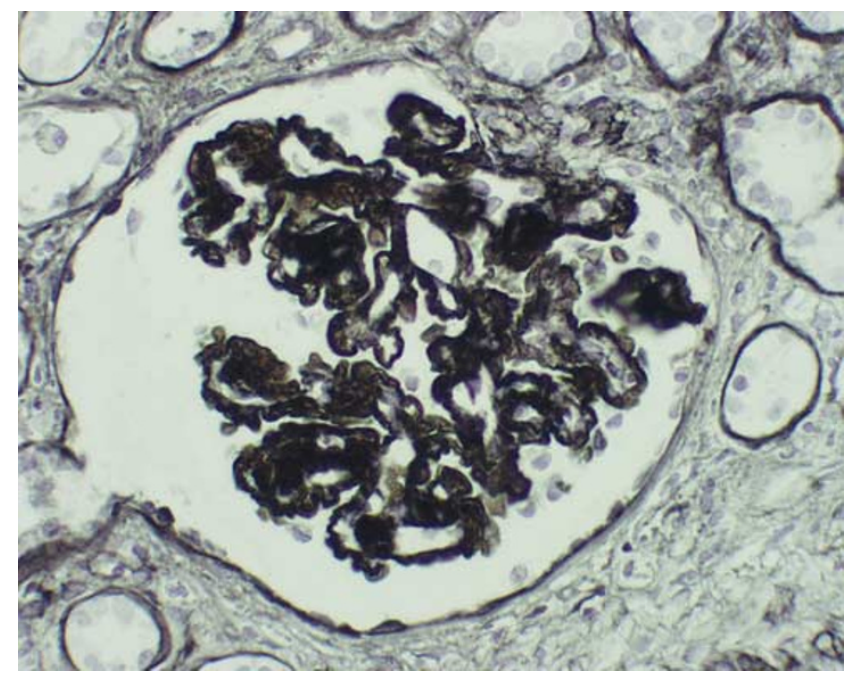

Figure 2 First biopsy. Immunohistochemistry on paraffin sections for kappa light chain (counterstained with hematoxylin) demonstrates strong staining of the glomerular capillary walls, mesangium, Bowman's capsule, and adjacent tubular basement membranes. Lambda light chain (not shown) shows no staining of similar renal structures.

of immunohistochemistry performed on the formalin-fixed, paraffin-embedded sections demonstrated strong kappa light chain staining for mesangial areas and the glomerular and tubular basement membranes (Figure 2), but IgG and lambda light chain were both negative. Electron microscopy of three representative glomeruli showed massive accumulations of electron-dense material in subendothelial regions of the peripheral capillary walls and in mesangial areas (Figure 3). The electrondense deposits lacked substructural organization.

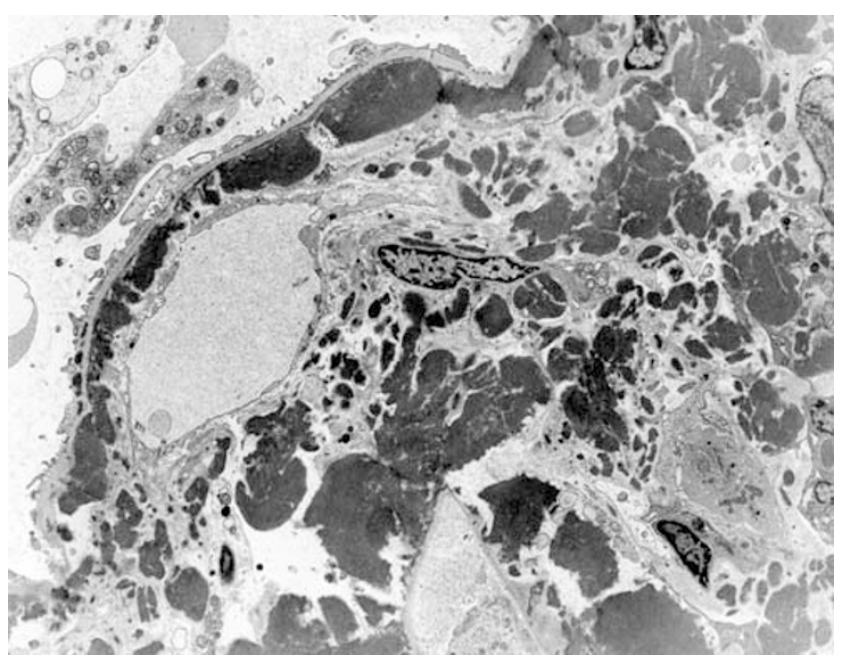

Figure 3 First biopsy. Massive electron-dense deposits are present along the subendothelial aspect of a peripheral capillary loop and in mesangial regions, corresponding to the eosinophilic material seen in Figure 1 (electron microscopy, original magnification $\times 6900$ ).

Endothelial tubuloreticular inclusions were not identified. Examination of the tubular basement membranes and interstitium showed no evidence of electron-dense deposits similar to those seen in the glomeruli.

\section{Clinical follow-up}

A bone marrow biopsy performed subsequent to the renal biopsy demonstrated 30\% plasma cells and both serum and urine immunoelectrophoresis and immunofixation electrophoresis revealed a monoclonal kappa light chain immunoglobulin. The amount of protein was not quantitated in the urine. No lytic bone lesions were identified by a skeletal X-ray survey. The patient received a regimen of melphalan and prednisone over the next 8-10 months. However, this regimen was halted after only three cycles due to numerous complications, including bleeding, thrombocytopenia, neutropenia, and several episodes of sepsis. During this period, she was anuric, but 3 months after the renal biopsy there was recommencement of urine production. At 2 months after this resumption of urine output, her creatinine clearance was $18 \mathrm{ml} / \mathrm{min}$ and total urine protein declined to $1.5 \mathrm{~g} / 24 \mathrm{~h}$. Dialysis was stopped shortly thereafter and the serum creatinine returned to a baseline of $1.0-1.2 \mathrm{mg} / \mathrm{dl}(88.4-106.1 \mu \mathrm{mol} / \mathrm{l})$. A repeat bone marrow biopsy at that time revealed $5 \%$ plasma cells. Several bone marrow biopsies over the next several years were negative for a plasma cell neoplasm, of which some were confirmed by flow cytometry. Regular testing of serum and urine immunofixation electrophoresis were negative for a monoclonal immunoglobulin. Of note, the patient developed overt diabetes mellitus approximately 2 years prior to the second renal biopsy. Her blood 
glucose and hemoglobin $\mathrm{A}_{1 \mathrm{C}}$ levels initially showed persistent elevation, but have subsequently normalized with administration of glyburide and diet control.

At 2 months prior to a second renal biopsy, a serum protein electrophoresis showed a new finding of hypogammaglobulinemia. At 1 month prior to this repeat biopsy, she developed lower extremity edema and her urinalysis demonstrated $3+$ protein. Laboratory tests revealed a serum creatinine of $1.7 \mathrm{mg} / \mathrm{dl}(150.3 \mu \mathrm{mol} / \mathrm{l})$ and albumin of $2.0 \mathrm{mg} / \mathrm{dl}$ $(20 \mathrm{~g} / \mathrm{l})$. Her dyspnea worsened and her headaches returned. A chest X-ray revealed pleural effusions, and an abdominal ultrasound showed normal sized kidneys, but ascites was present. The 24-h urine showed a creatinine clearance of $34 \mathrm{ml} / \mathrm{min}$ $(0.57 \mathrm{ml} / \mathrm{s})$ and total protein excretion of $13.5 \mathrm{~g}$. Subsequent serologic tests for ANA were negative and serum complement levels were normal. At 83 months after the initial renal biopsy, a second renal biopsy was performed. Both serum and urine immunofixation electrophoresis studies demonstrated a monoclonal kappa light chain immunoglobulin.

\section{Renal biopsy \#2 pathology}

The second biopsy contained 36-58 glomeruli per level section for light microscopic evaluation. Approximately $30 \%$ of these glomeruli were globally sclerotic. The remaining glomeruli demonstrated massive accumulations of eosinophilic material in mesangial regions and in subendothelial portions of glomerular capillary walls similar to the first biopsy (Figure 4). There was accentuation of the lobular architecture due to the massive accumulations of eosinophilic material, but nodular glomerulosclerosis and mesangial hypercellularity were not prominent features. Glomerular inflammatory cell influx, crescent formation, or necrosis of glomerular tufts was not identified. The tubulointerstitial compartment showed patchy, mild interstitial fibrosis and tubular atrophy. The arteries showed moderate intimal sclerosis and the arterioles demonstrated frequent subendothelial hyalinosis. A Congo red stain was negative for amyloid. Immunofluorescence microscopy of 14 glomeruli revealed diffuse and strong staining $(4+/ 4+)$ of the glomeruli in mesangial areas and glomerular basement membranes for kappa but not lambda light chains (Figure 5a). The tubular basement membranes and interstitium showed patchy and strong staining for kappa light chains $(4+/ 4+$ ) (Figure $5 b$ ), but not lambda light chains. C3 stained the glomeruli diffusely similar to kappa light chains but less intensely $(2-3+/ 4+)$. There was weak (less than 1 $+/ 4+)$ and segmentally distributed linear staining of the glomerular basement membranes for IgG. There was no significant glomerular staining for IgA, IgM, C1q, lambda light chains, fibrinogen, or

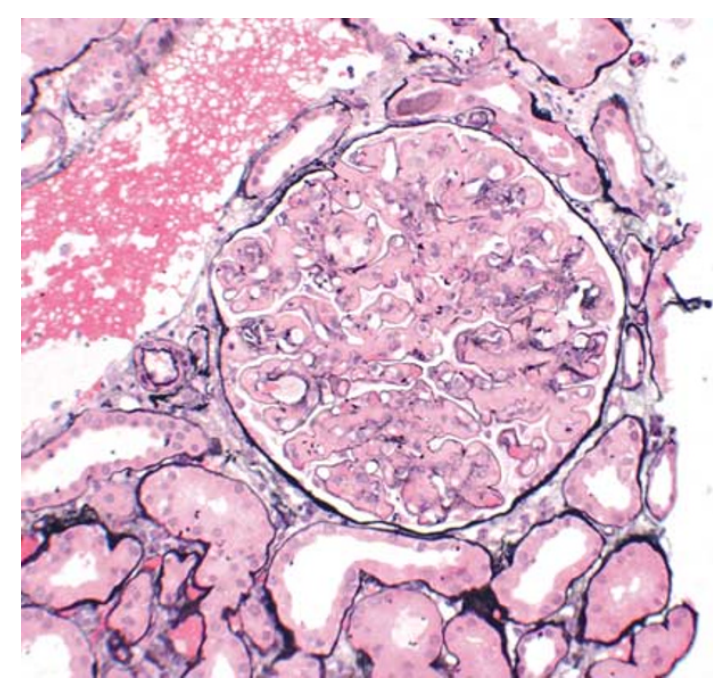

1001

Figure 4 Second biopsy. This glomerulus exhibits massive accumulations of amorphous eosinophilic material in subendothelial regions, identical to the first biopsy (Jones silver methenamine).

albumin. Ultrastructural studies demonstrated massive accumulation of electron-dense deposits identical in appearance to those seen in the first biopsy in subendothelial locations along the glomerular basement membranes and more focally in subepithelial locations as well. These deposits extended prominently into mesangial areas. Examination of the interstitium and peritubular capillaries showed occasional aggregates of similar electron-dense deposits (Figure 6). The tubular basement membranes revealed ill-defined electron-dense material, which could represent either cellular debris or immune complexes. The final diagnosis was light chain deposition disease, of monoclonal kappa light chain type.

\section{Clinical follow-up \#2}

A bone marrow biopsy around the time of the second renal biopsy revealed a clonal kappa light chain restricted plasma cell neoplasm, which was confirmed by flow cytometry. There remained no lytic bone lesions visualized by MRI. She was being treated with thalidomide and doing well with the exception of some neuropathy. At 7 months after the second renal biopsy, her serum albumin was $4.1 \mathrm{mg} / \mathrm{dl}(41 \mathrm{~g} / \mathrm{l})$ and a spot urine protein decreased to $285.5 \mathrm{mg} / \mathrm{dl}$ with a urine creatinine of $41 \mathrm{mg} / \mathrm{dl}$. Her serum creatinine was approximately $2.7 \mathrm{mg} / \mathrm{dl}$ $(238.7 \mu \mathrm{mol} / \mathrm{l})$, and she remained on hemodialysis (2 days per week).

\section{Discussion}

Light chain deposition disease is the most common entity under the broad classification of monoclonal immunoglobulin deposition disease, which also 

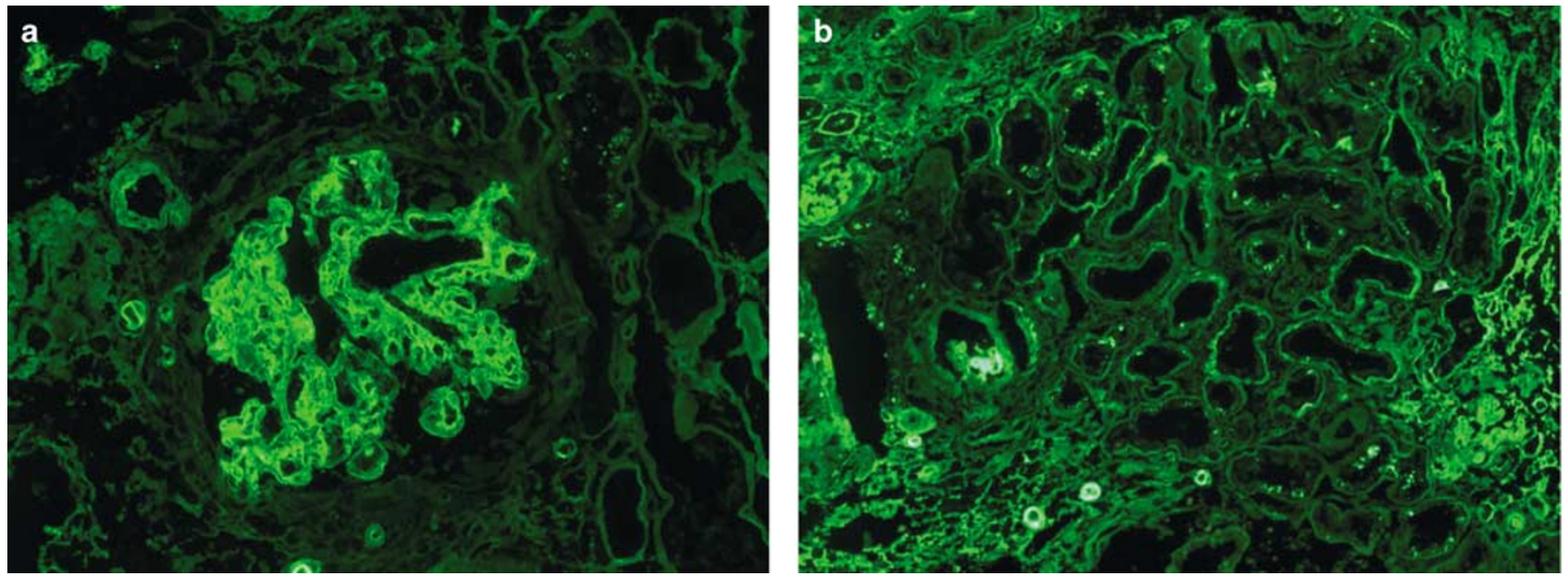

Figure 5 Immunofluorescence microscopy of the second biopsy. (a) A representative glomerulus shows diffuse and strong confluent kappa light chain staining of the glomerular capillary walls and mesangium. There is no significant staining of Bowman's capsule, tubular basement membranes in this region, unlike the region depicted in (b). No staining for lambda light chains was observed in the glomeruli, tubular basement membranes, or interstitium (not shown). (b) There is patchy and strong kappa light chain staining of the tubular basement membranes and interstitium.

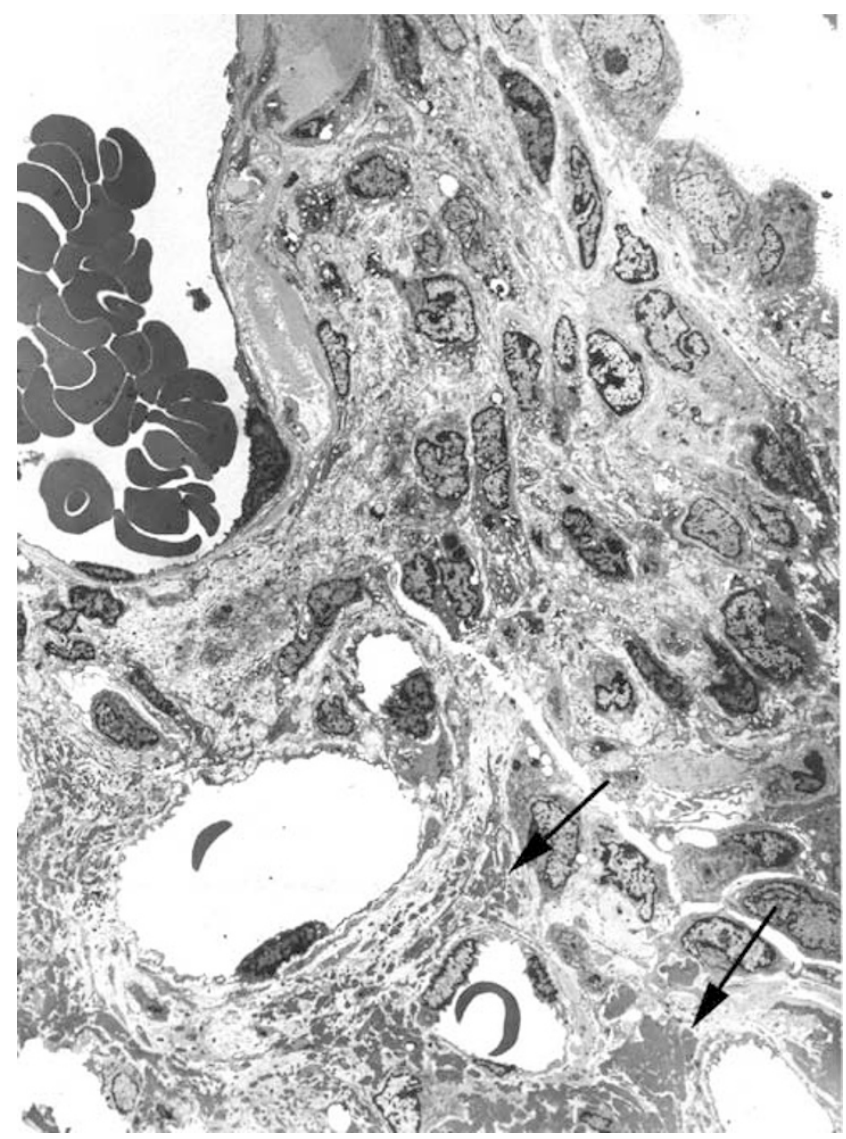

Figure 6 Second biopsy. There are prominent aggregates of electron-dense deposits focally present in the interstitium (arrows point to some of the many deposits present). Glomerular deposits identical to those seen in Figure 3 were uniformly present but not illustrated in this field (electron microscopy, original magnification $\times 2600$ )

consists of light and heavy chain deposition disease and heavy chain deposition disease. ${ }^{12,13}$ It has been over 30 years since the early descriptions of light chain deposition disease in 1973 by Antonovych et $a 1^{14}$ with additional characterization by Randall et $a l^{4}$ in 1976. Light chain deposition disease is thought to affect $3-5 \%$ of patients with underlying multiple myeloma or other plasma cell dyscrasia. ${ }^{15,16}$ In $30 \%$ of light chain deposition disease cases, there is an absence of an underlying plasma cell dyscrasia or lymphoproliferative disorder by histologic examination despite the presence of a monoclonal gammopathy. ${ }^{17}$

The most common glomerular histopathological lesion in light chain deposition disease is nodular glomerulosclerosis, which is present in $55-100 \%$ of renal biopsies within some series. ${ }^{1,3,13}$ The histologic spectrum of light chain deposition disease can be quite variable, and ranges from minimal glomerular changes with or without glomerular deposits to membranoproliferative or mesangial proliferative glomerulonephritis. ${ }^{2-9}$ These lesions can occur independently of nodular glomerulosclerosis but often occur concomitantly with nodular glomerulosclerosis in the same biopsy. All of these histologic subtypes can occur with or without crescents. ${ }^{5}$

Since the first description of light chain deposition disease, over 150 cases have been documented in the English language medical literature. ${ }^{2-7,10,18-23}$ Despite the common occurrence of light chain deposition disease, our current case represents a unique manifestation of light chain deposition disease with histologic and ultrastructural features that resemble a massive immune-complex deposition form of glomerulonephritis, which has not been previously reported to our knowledge. Nodular accentuation of the glomerular tuft architecture was present focally in both biopsies, but this feature was overshadowed by the prominent accumulation of amorphous eosinophilic material corresponding to the prominent electron-dense deposits involving all mesangial regions and glomerular capillary loops. 
The light microscopic findings considered in isolation were most suggestive of an immunecomplex-mediated glomerulonephritis, such as lupus nephritis. The prominent eosinophilic material in the subendothelial aspects of the glomerular basement membranes most closely resembled the 'wire-loop' deposits of focal or diffuse proliferative glomerulonephritis in systemic lupus erythematosus. In fact, the constellation of histologic and ultrastructural findings in conjunction with the positive ANA titer and the absence of immunofluorescence microscopy led to an initial provisional diagnosis of lupus nephritis in this patient's initial biopsy. However, the clinical presentation and the absence of endothelial tubuloreticular inclusions detectable by electron microscopy argued against the diagnosis of lupus nephritis at that time. The patient's proteinuria and abnormal ANA titer satisfied only two of four criteria in the 1982 American Rheumatism Association criteria for the classification of lupus that were necessary to establish a diagnosis. Subsequent immunohistochemistry of the first biopsy and immunofluorescence microscopy of the second biopsy confirmed the absence of IgA, IgM, C1q, or significant levels of IgG deposition and demonstrated strong staining only for kappa light chains, thereby establishing the diagnosis of light chain deposition disease.

Weak segmental and linear staining of some of the glomerular basement membranes for IgG by immunofluorescence microscopy was also present. We believe this pattern of weak, linear IgG staining, distinct from the strong, diffuse and confluent staining for kappa light chain, most likely represents a nonspecific finding consequent to altered affinities of the damaged capillary wall similar to that frequently observed in diabetic nephropathy and some allograft kidneys. In view of the patient's 2year history of diabetes, it is likely that the $\operatorname{IgG}$ staining is a manifestation of the weak linear staining of glomerular capillary walls for IgG that is commonly encountered in renal biopsies of patients with diabetes mellitus. In support of this interpretation, the large glomerular and interstitial deposits, tubular basement membranes, and interstitium were noticeably negative for IgG but strongly positive for kappa light chains by immunofluorescence microscopy. Finally, this patient's urine or serum protein immunoelectrophoresis and immunofixation electrophoresis studies have detected only kappa light chains but not an IgG component of the monoclonal immunoglobulin.

This case is of particular interest in view of a series of cases recently reported by Nasr et al, ${ }^{10}$ describing a purportedly new clinicopathologic entity with essentially identical ultrastructural features to our current case. ${ }^{10}$ They collected 10 cases that demonstrated monoclonal IgG deposits with histologic and ultrastructural features mimicking an immune-complex glomerulonephritis. Notably, the immunoglobulin deposition involved only the glomeruli and not the interstitium or tubular basement membranes. Five of the patients had a detectable monoclonal protein by either serum or urine protein electrophoresis, but despite extensive workups, none of these patients had additional evidence of an underlying multiple myeloma or plasma cell dyscrasia. In their report, Nasr et al reviewed the literature and found at least four additional cases which demonstrated light and heavy chain deposition disease and massive immune-complex type deposits in patients without an underlying hematopoietic disorder. ${ }^{7,24-26}$

Our case is noteworthy for its expansion of the spectrum of clinicopathologic findings that may be encountered in glomerulopathies attributable to a monoclonal immunoglobulin deposition disorder but whose pathologic features mimic a proliferative glomerulonephritis of immune-complex type. First, our patient had a 7-year history of multiple myeloma, well documented by bone marrow biopsy, in contrast to the patients of Nasr et $a l^{10}$ who lacked evidence of overt neoplasia. Second, immunofluorescence studies demonstrated a light chain deposition process, with a corresponding circulating monoclonal kappa chain detectable by serum and urine immunoelectrophoresis and immunofixation studies, rather than a monoclonal immunoglobulin composed of both light and heavy chains. Finally, the deposition process in the present case was not limited to the glomeruli, but also involved the tubular basement membranes and interstitium. In our review of the literature, we have identified one case of light and heavy chain deposition disease with a documented history of myeloma, which demonstrated prominent granular electron-dense deposits, in addition to those cases with such deposits that have been reported previously or reviewed by Nasr et al. ${ }^{10,27}$ Although the histologic features in this last case showed nodular glomerulosclerosis rather than a proliferative glomerulonephritis, we believe that all of these reported cases, including our present case, represent the wide histologic spectrum that can be encountered within the broad category of monoclonal immunoglobulin deposition disease.

Although based on a single case, our clinical follow-up data of 7 years indicates that this variant may represent a less aggressive form of light chain deposition disease, which typically has a median patient survival and renal survival time of 49-69 months and 2 years, respectively. ${ }^{13,17}$

In summary, this report illustrates an unusual clinical and histologic presentation of light chain deposition disease, further expanding the histologic spectrum that is already recognized within the categories of monoclonal immunoglobulin deposition disease and, specifically, light chain deposition disease. Although the histologic features are unusual, the combination of clinical information with the morphologic evaluation established the diagnosis of light chain deposition disease. The 7-year 
clinical follow-up suggests that this variant may have a less aggressive clinical course than the usual form of light chain deposition disease, but additional follow-up of this and other cases are necessary to establish the clinical significance of this rare variant.

\section{References}

1 Paueksakon P, Revelo MP, Horn RG, et al. Monoclonal gammopathy: significance and possible causality in renal disease. Am J Kidney Dis 2003;42:87-95.

2 Strom EH, Fogazzi GB, Banfi G, et al. Light chain deposition disease of the kidney. Morphological aspects in 24 patients. Virchows Arch 1994;425: 271-280.

3 Sanders PW, Herrera GA, Kirk KA, et al. Spectrum of glomerular and tubulointerstitial renal lesions associated with monotypical immunoglobulin light chain deposition. Lab Invest 1991;64:527-537.

4 Randall RE, Williamson Jr WC, Mullinax F, et al. Manifestations of systemic light chain deposition. Am J Med 1976;60:293-299.

5 Venkataseshan VS, Faraggiana T, Hughson MD, et al. Morphologic variants of light-chain deposition disease in the kidney. Am J Nephrol 1988;8:272-279.

6 Ivanyi B, Varga G, Nagy J, et al. Light chain deposition nephropathy in necropsy material. Zentralbl Pathol 1991;137:366-371.

7 Alpers CE, Tu WH, Hopper Jr J. Single light chain subclass (kappa chain) immunoglobulin deposition in glomerulonephritis. Hum Pathol 1985;16:294-304.

8 Silva FG, Meyrier A, Morel-Maroger L, et al. Proliferative glomerulonephropathy in multiple myeloma. J Pathol 1980;130:229-236.

9 Ganeval D, Cathomen M, Noel LH, et al. Kidney involvement in multiple myeloma and related disorders. Contrib Nephrol 1982;33:210-222.

10 Nasr SH, Markowitz GS, Stokes MB, et al. Proliferative glomerulonephritis with monoclonal IgG deposits: A distinct entity mimicking immune-complex glomerulonephritis. Kidney Int 2004;65:85-96.

11 Grogan TM, Van Camp B, Kyle RA, et al. Plasma cell neoplasms In: Jaffe ES, Harris NL, Stein H, Vardiman JW (eds) WHO Classification of Tumours. Pathology and Genetics of Haematopoietic and Lymphoid Tissues. IARC Press: Lyon, 2001, pp 142-156.

12 Gallo G, Picken M, Buxbaum J, et al. The spectrum of monoclonal immunoglobulin deposition disease associated with immunocytic dyscrasias. Semin Hematol 1989;26:234-245.

13 Lin J, Markowitz GS, Valeri AM, et al. Renal monoclonal immunoglobulin deposition disease: the disease spectrum. J Am Soc Nephrol 2001;12:1482-1492.
14 Antonovych T, Line C, Parrish E, et al. Light chain deposits in multiple myeloma. Lab Invest 1974;30: 730A (abstract).

15 Ivanyi B. Frequency of light chain deposition nephropathy relative to renal amyloidosis and Bence Jones cast nephropathy in a necropsy study of patients with myeloma. Arch Pathol Lab Med 1990; 114:986-987.

16 Herrera GA, Joseph L, Gu X, et al. Renal pathologic spectrum in an autopsy series of patients with plasma cell dyscrasia. Arch Pathol Lab Med 2004;128: 875-879.

17 Pozzi C, D’Amico M, Fogazzi GB, et al. Light chain deposition disease with renal involvement: clinical characteristics and prognostic factors. Am J Kidney Dis 2003;42:1154-1163.

18 Buxbaum JN, Chuba JV, Hellman GC, et al. Monoclonal immunoglobulin deposition disease: light chain and light and heavy chain deposition diseases and their relation to light chain amyloidosis. Clinical features, immunopathology, and molecular analysis. Ann Intern Med 1990;112:455-464.

19 Gallo GR, Feiner HD, Katz LA, et al. Nodular glomerulopathy associated with nonamyloidotic kappa light chain deposits and excess immunoglobulin light chain synthesis. Am J Pathol 1980;99:621-644.

20 Bangerter AR, Murphy WM. Kappa light chain nephropathy. A pathologic study. Virchows Arch A Pathol Anat Histopathol 1987;410:531-539.

21 Tubbs RR, Gephardt GN, McMahon JT, et al. Light chain nephropathy. Am J Med 1981;71:263-269.

22 Preud'Homme JL, Morel-Maroger L, Brouet JC, et al. Synthesis of abnormal heavy and light chains in multiple myeloma with visceral deposition of monoclonal immunoglobulin. Clin Exp Immunol 1980;42: $545-553$.

23 Seymour AE, Thompson AJ, Smith PS, et al. Kappa light chain glomerulosclerosis in multiple myeloma. Am J Pathol 1980;101:557-580.

24 Jokiranta TS, Solomon A, Pangburn MK, et al. Nephritogenic lambda light chain dimer: a unique human miniautoantibody against complement factor H. J Immunol 1999;163:4590-4596.

25 Preud'homme JL, Aucouturier P, Touchard G, et al. Monoclonal immunoglobulin deposition disease (Randall type). Relationship with structural abnormalities of immunoglobulin chains. Kidney Int 1994;46: 965-972.

26 Bridoux F, Binaut R, Zanetta G, et al. Glomerulopathy with non-organized and non-Randall type monoclonal immunoglobulin deposits: a rare entity. J Am Soc Nephrol 2001;12:94A (abstract).

27 Chang IJ, Cheunsuchon B, Miller FJ, et al. A 66-yearold woman with progressive renal insufficiency, nephrotic syndrome, and monoclonal protein. Am J Kidney Dis 2003;41:508-517. 